NASA/TM-2003-212664

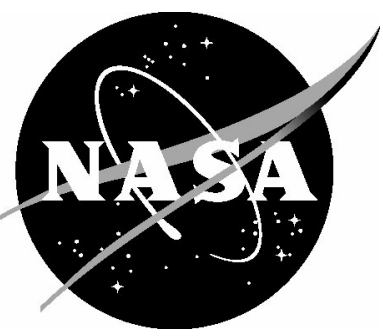

\title{
A Reduced-Order Model for Efficient Simulation of Synthetic Jet Actuators
}

Nail K. Yamaleev

North Carolina A\&T State University, Greensboro, North Carolina

Mark H. Carpenter

Langley Research Center, Hampton, Virginia 
Since its founding, NASA has been dedicated to the advancement of aeronautics and space science. The NASA Scientific and Technical Information (STI) Program Office plays a key part in helping NASA maintain this important role.

The NASA STI Program Office is operated by Langley Research Center, the lead center for NASA's scientific and technical information. The NASA STI Program Office provides access to the NASA STI Database, the largest collection of aeronautical and space science STI in the world. The Program Office is also NASA's institutional mechanism for disseminating the results of its research and development activities. These results are published by NASA in the NASA STI Report Series, which includes the following report types:

- TECHNICAL PUBLICATION. Reports of completed research or a major significant phase of research that present the results of NASA programs and include extensive data or theoretical analysis. Includes compilations of significant scientific and technical data and information deemed to be of continuing reference value. NASA counterpart of peerreviewed formal professional papers, but having less stringent limitations on manuscript length and extent of graphic presentations.

- TECHNICAL MEMORANDUM. Scientific and technical findings that are preliminary or of specialized interest, e.g., quick release reports, working papers, and bibliographies that contain minimal annotation. Does not contain extensive analysis.

- CONTRACTOR REPORT. Scientific and technical findings by NASA-sponsored contractors and grantees.
- CONFERENCE PUBLICATION. Collected papers from scientific and technical conferences, symposia, seminars, or other meetings sponsored or co-sponsored by NASA.

- SPECIAL PUBLICATION. Scientific, technical, or historical information from NASA programs, projects, and missions, often concerned with subjects having substantial public interest.

- TECHNICAL TRANSLATION. Englishlanguage translations of foreign scientific and technical material pertinent to NASA's mission.

Specialized services that complement the STI Program Office's diverse offerings include creating custom thesauri, building customized databases, organizing and publishing research results ... even providing videos.

For more information about the NASA STI Program Office, see the following:

- Access the NASA STI Program Home Page at http://www.sti.nasa.gov

- E-mail your question via the Internet to help@sti.nasa.gov

- Fax your question to the NASA STI Help Desk at (301) 621-0134

- Phone the NASA STI Help Desk at (301) 621-0390

- Write to: NASA STI Help Desk NASA Center for AeroSpace Information 7121 Standard Drive Hanover, MD 21076-1320 
NASA/TM-2003-212664

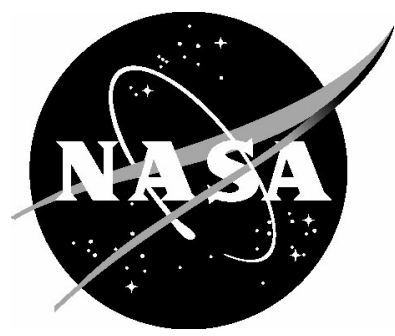

\section{A Reduced-Order Model for Efficient Simulation of Synthetic Jet Actuators}

Nail K. Yamaleev

North Carolina A\&T State University, Greensboro, North Carolina

Mark H. Carpenter

Langley Research Center, Hampton, Virginia

National Aeronautics and

Space Administration

Langley Research Center

Hampton, Virginia 23681-2199

December 2003 
Available from:

NASA Center for AeroSpace Information (CASI)

7121 Standard Drive

Hanover, MD 21076-1320

(301) 621-0390
National Technical Information Service (NTIS) 5285 Port Royal Road Springfield, VA 22161-2171

(703) 605-6000 


\title{
A Reduced-Order Model for Efficient Simulation of Synthetic Jet Actuators
}

\author{
Nail K. Yamaleev ${ }^{a}$ and Mark H. Carpenter ${ }^{b}$ \\ ${ }^{a}$ Department of Mathematics, North Carolina A\&T State University \\ ${ }^{\mathrm{b}}$ NASA Langley Research Center
}

\begin{abstract}
A new reduced-order model of multidimensional synthetic jet actuators that combines the accuracy and conservation properties of full numerical simulation methods with the efficiency of simplified zero-order models is proposed. The multidimensional actuator is simulated by solving the time-dependent compressible quasi-1-D Euler equations, while the diaphragm is modeled as a moving boundary. The governing equations are approximated with a fourth-order finite difference scheme on a moving mesh such that one of the mesh boundaries coincides with the diaphragm. The reduced-order model of the actuator has several advantages. In contrast to the 0-D models, this approach provides conservation of mass, momentum, and energy. Furthermore, the new method is computationally much more efficient than the multidimensional Navier-Stokes simulation of the actuator cavity flow, while providing practically the same accuracy in the exterior flowfield. The most distinctive feature of the present model is its ability to predict the resonance characteristics of synthetic jet actuators; this is not practical when using the 3-D models because of the computational cost involved. Numerical results demonstrating the accuracy of the new reduced-order model and its limitations are presented.
\end{abstract}

Key words: reduced-order model, synthetic jet actuator, active flow control, high-order finite difference method, Navier-Stokes equations, quasi-1-D Euler equations

\section{Introduction}

Interest in active flow control for drag or noise reduction, flow vectoring, mixing enhancement, and separation control stimulated the recent development of innovative synthetic jet actuators that create localized disturbances in a flowfield. Synthetic jets are generated by a dynamic fluid actuator consisting of a cavity enclosed on one side by a moving diaphragm driven into transverse 


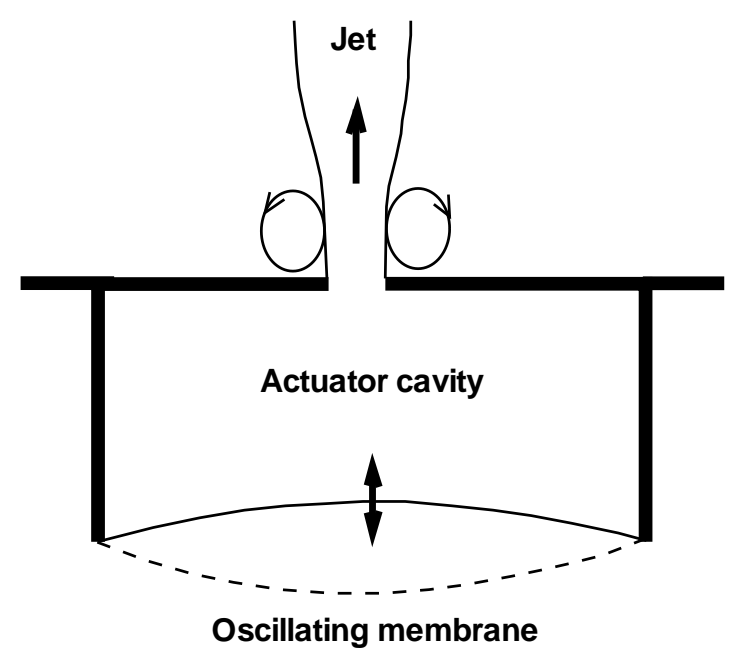

Fig. 1. Schematic of a synthetic jet actuator.

oscillations at its resonance frequency and on the other side by a rigid wall with a small orifice or slot, as shown in Fig. 1. The distinctive feature of these actuators is that they have minimal power requirements and jet-like characteristics without the need for net mass injection. Although there is no net mass injection into the overall system over each cycle of operation, the momentum transfer into the embedding flow is nonzero. These features enable synthetic jets to effect significant global modifications in the embedding flow on scales that are one to two orders of magnitude larger than the characteristic length or force scale of the jets themselves [1].

Accurate details of the general performance of fluid actuators is desirable over a range of flow conditions, within some predetermined error tolerance. Designers typically model actuators with different levels of fidelity depending on the acceptable level of error in each circumstance. Crude properties of the actuator (e.g., peak mass rate and frequency) may be sufficient for some designs, while detailed information is needed for other applications (e.g., multiple actuator interactions).

This work attempts to address two primary objectives. The first objective is to develop a systematic methodology for approximating realistic 3-D fluid actuators, using quasi-1-D reduced-order models. Near full fidelity can be achieved with this approach at a fraction of the cost of full simulation and only a modest increase in cost relative to most actuator models used today. Specific considerations include 1) a general statement on which model geometries are amenable to the quasi-1-D approximation, 2) regions of actuators that are inherently multidimensional and are not candidates for reduced-order modeling, and 3) the geometric features within actuators that contribute little to the fidelity of the actuator solution. The second objective, which is a direct consequence of the first, is to determine the approximate magnitude of errors committed by 
actuator model approximations of various fidelities. This objective attempts to identify which model (ranging from simple orifice exit boundary conditions to full numerical simulations of the actuator) is appropriate for a given error tolerance.

The numerical simulation and optimization of synthetic jet actuators has received considerable attention [2-14]. The exact nature of any actuator can be determined (in principle) by simulating the precise 3-D geometry, including all aspects of diaphram movement and deformation. The separation of temporal and spatial scales, and moving boundaries in the problem makes full computational simulation of these unsteady flows impractical. A wide range of various fidelity simplified models have been developed. Assuming various characteristics of the actuator can significantly reduce the simulation cost of the actuator. The extreme opposite full simulation is characterized by imposing experimental data at the actuator orifice. If reliable experimental data is available, then this approach is desirable. All general actuator models, however, fall somewhere between these two extremes, and attempt to trade fidelity for cost in some measure. A brief summary of the popular methods is now presented.

One class of models attempts to impose simplified boundary conditions at the orifice exit, by directly prescribing the harmonic motion generated by the actuator. In the work of Kral et al. [2] and Donovan, Kral, and Cary [3], the flow within the cavity is not calculated and the perturbation to the flowfield is introduced through the wall-normal component of velocity at the orifice exit

$$
\begin{aligned}
& v(x, 0, t)=V_{A}(x) \sin (\omega t) \\
& u(x, 0, t)=0
\end{aligned}
$$

where $x$ and $y$ are the flow streamwise and cross-stream directions, respectively. Different spatial profiles of $V_{A}(x)$ over the orifice have been considered. Numerical experiments [2,3] (within the scope of their model) indicate that a "top-hat" distribution most closely matches the experimental data. A modified boundary condition on the pressure at the orifice is introduced through a consideration of the normal momentum equation. Taking into account timeharmonic velocity perturbations, this condition, obtained under the assumption that the flow is incompressible, becomes

$$
\frac{\partial p}{\partial y}=-\rho V_{A}(x) \omega \cos (\omega t)
$$

The numerical simulations using the boundary conditions given by Eqs. (1)(2) show good qualitative agreement with the experiments in Refs. $[1,15,16]$. A similar approach modeling the actuator as a blowing/suction type boundary 
condition, which can be fully specified in advance of the calculation, is used in Refs. [4-7].

An alternative technique for modeling synthetic jet actuators is proposed by Carpenter, Lockerby, and Davies [8]. This actuator model is based on the classic thin plate theory for the diaphragm dynamics. The flow through the orifice is modeled by using the unsteady pipe-flow theory. This approach is based on the assumption that streamlines in the orifice exit are parallel to its axis, which is an adequate approximation if the length-to-diameter ratio is much larger than unity. The governing equation for the orifice flow is

$$
\rho \frac{\partial v}{\partial t}+\frac{\rho v|v|}{2 l}=-\frac{\partial p}{\partial y}+\mu r \frac{\partial}{\partial r}\left(\frac{1}{r} \frac{\partial v}{\partial r}\right)
$$

where $y$ and $r$ are the axial and radial coordinates, respectively, $v$ is the axial velocity, $l$ is the orifice length, and $\rho$ is taken to be the instantaneous mean of the cavity and external densities. The inertial term $\rho v v_{y}$ is modeled approximately by the second term on the left-hand side of Eq. (3). The dynamics of the air in the cavity is ignored and the pressure there is calculated by means of the perfect gas law. The cavity and the external boundary layer flowfields are linked by requiring continuity of velocity and pressure at the orifice exit. In Ref. [8], only the blowing phase of the actuator dynamics is studied.

A 0-D model based on a system of ordinary differential equations describing a resonant fluid actuator that creates an unsteady synthetic jet through compression of fluid in a cavity is developed in Ref. [9]. Assuming that the flow is isothermal and combining structural and fluid effects, a set of five coupled nonlinear first-order ordinary differential equations, including the membrane position and velocity, the fluid density and pressure, and the jet velocity, is derived. This 0-D model is compared with experimental data and successfully predicts trends in the actuator behavior.

A similar reduced-order simulation of a piezoelectric-driven synthetic jet actuator using a lumped element model is performed in Ref. [10]. In lumped element modeling, the individual components of the actuator are modeled as elements of an electrical circuit by using conjugate power variables. The linear composite plate theory is used to calculate the net volume velocity of the diaphragm and the distributed vertical velocity. The acoustic mass and resistance in the orifice are obtained by assuming fully developed laminar pipe flow in the actuator neck. The model is applied to two prototypical synthetic jets and provides fair agreement with the measured peak jet velocity. As follows from this study, the lumped element model does not provide the dependance of the actuator behavior on the actuator geometry and neglects nonlinear effects due to large amplitude pressure oscillations inside the cavity and compressibility effects in the orifice. Therefore, better models are needed for the unsteady 
flow in the orifice, including that of entrance and exit effects.

A second class of methods is based on a direct numerical simulation of the entire problem, including the flow inside the actuator. Rizzetta, Visbal, and Stanek [11] solve the unsteady compressible Navier-Stokes equations in the external region, the cavity itself, and the throat of the actuator on separate grids that are linked with each other through a Chimera methodology. The membrane motion is simulated by varying the position of appropriate boundary points. As follows from the numerical calculations presented in Ref. [11], the internal cavity flow becomes periodic after several cycles of diaphragm oscillation. Therefore, the velocity profile across the jet exit at each time step was recorded and was used as a boundary condition in subsequent runs involving the external domain only. For computations that consider only the upper exterior domain, the transverse and spanwise velocity components (orthogonal to jet axis) are set to zero, and the inviscid normal momentum equation

$$
\frac{\partial p}{\partial y}=-\rho\left(\frac{\partial v}{\partial t}+v \frac{\partial v}{\partial y}\right)
$$

is used to establish the pressure. The density at the orifice exit is extrapolated from the interior solution. This approach provides a more accurate description of the flow details at the orifice than the simplified boundary conditions given by Eqs. (1)-(2). Similar direct numerical simulation of the external and cavity flows is performed by Joslin, Lachowicz, and Yao [12] and shows good agreement with experimental results.

Another approach that simulates the diaphragm as a moving boundary is used in Ref. [13]. This technique simulates the entire actuator geometry, including the oscillating diaphragm, on a stationary Cartesian mesh. As the diaphragm moves over the underlying Cartesian mesh, the discretization in the cell cut by the solid boundary is modified to account for the presence of the diaphragm. Thus, the grid does not need to conform to the moving boundary, which simplifies the gridding of the deforming domain. This approach is applied to study the vortex dynamics, velocity profiles, and other dynamical characteristics of the incompressible low-Reynolds-number synthetic jet flow.

To avoid the integration of the Navier-Stokes equations on a moving grid, an alternative technique is used by Lee and Goldstein [14]. Their method imposes a localized body force along desired points in the computational mesh to bring the fluid there to a specified velocity so that the force has the same effect as a solid boundary. The desired velocity is incorporated in an iterative feedback loop to determine the appropriate force. For a moving boundary with velocity 
$V_{A}(x, t)$, an expression for the body force is given by

$$
F(x, t)=\alpha \int_{0}^{t}\left(v-V_{A}\right) d t^{\prime}+\beta\left(v-V_{A}\right),
$$

where $v$ is the fluid velocity, and $\alpha$ and $\beta$ are user-defined constants that are negative and can be treated as the gain and damping of the force field. This approach simulates the moving diaphragm without using a time-dependent coordinate transformation.

Although the methods mentioned above have successfully been used for modeling synthetic jet actuators, several issues persist. The principle difficulty inherent in the 0-D models based on Eqs. (1)-(4) is their inability to predict accurate pressure levels in the actuator. This difficulty is extremely apparent in the suction phase of the cycle, where pressure is the variable that determines the influx of mass. Imposing the incorrect pressure during this phase will lead to erroneous results. The primary reason 0-D models "drift" away from the correct solution is that they do not conserve mass, momentum, or energy through the actuator orifice. Because these methods use the normal momentum equation to calculate the pressure, whereas the other quantities are extrapolated or prescribed analytically, these boundary conditions do not satisfy the governing equations at the boundary and, therefore, do not provide conservation properties. The $0-\mathrm{D}$ boundary conditions lack a mechanism to account for changes in the pressure field caused by the interaction of the external boundary layer and the actuator. Furthermore, as shown in Ref. [14], the real streamwise velocity profile and the velocity component in the crossflow direction are far from the analytical expressions of Eq. (1).

The main problem associated with the full simulation methods is computational cost. The numerical calculation of the cavity flow requires significant computational resources, sometime comparable with that needed to resolve the exterior flow. For geometries fitted with multiple actuators, grid requirements for the actuators could considerably exceed those of the exterior flow, and would contribute extensively to the computational cost. The large range of temporal scales from $10^{-6} s$ to $10^{-3} s$, which are associated with the acoustic scale and the period of diaphragm oscillations, makes this problem even more complicated. Another consideration is the actuator Mach number, which varies from $O\left(10^{-3}\right)$ (near the diaphragm) to $O\left(10^{-1}\right)$ (at the orifice exit). This variation of the flow parameters from the fully incompressible to fully compressible regimes imposes severe requirements on a numerical method and increases the algorithm complexity.

As follows from the literature overview presented above, the research in the area of active flow control is of empirical nature, mainly due to the com- 
putational cost involved and lack of confidence in computational methods for such complex time-dependent flows. To overcome these problems, a new methodology that combines the accuracy and conservation properties of the full simulation methods with the efficiency of the techniques based on simplified boundary conditions is proposed. In contrast to the methods found in the literature, the new approach uses a reduced-order model to approximate the 2-D or 3-D synthetic jet actuators. The multidimensional actuator is simulated by solving the time-dependent 1-D Euler equations similar to those used for the quasi-1-D nozzle problem. The quasi-1-D governing equations are written in a time-dependent coordinate frame, and the diaphragm is modeled as a moving boundary. The low-dimensional actuator model has several advantages. First, in contrast to the 0-D models, this approach provides conservation of not only mass, but also momentum and energy. Second, the new method is much more efficient in terms of computational time compared with the full 2-D or 3-D numerical simulation techniques. Third, the reduced-order model retains some multidimensional features of the realistic actuator; these features are governed by the frequency and deflection of the diaphragm and the area variation and length of the quasi-1-D nozzle. Therefore, the proposed reducedorder model can be used for quantitative study of the resonance characteristics of the actuator.

The paper is organized as follows. Section 2 describes the governing equations and boundary conditions. Section 3 presents the the new reduced-order model of synthetic jet actuators. A fourth-order finite difference method used to solve both the interior and exterior flows is given in Section 4. Section 5 presents the numerical results and addresses accuracy, advantages, and limitations of the new model as well as its ability to predict the resonance characteristics of synthetic jet actuators. In Section 6, the questions that will be addressed in the future are discussed. Conclusions are summarized in Section 7.

\section{Governing Equations and Boundary Conditions}

The time-dependent 2-D Navier-Stokes equations are used to describe the unsteady compressible flow generated by a synthetic jet actuator. The governing equations in curvilinear coordinates $(\xi, \eta)$ can be written in conservation law form as

$$
\frac{\partial \hat{\mathbf{U}}}{\partial \tau}+\frac{\partial\left(\hat{\mathbf{F}}-\hat{\mathbf{F}}_{v}\right)}{\partial \xi}+\frac{\partial\left(\hat{\mathbf{G}}-\hat{\mathbf{G}}_{v}\right)}{\partial \eta}=0
$$


where the vector of conservative variables and the inviscid and viscous fluxes are given by

$$
\begin{aligned}
& \hat{\mathbf{U}}=\frac{1}{J} \mathbf{U}, \quad \hat{\mathbf{F}}=\frac{1}{J}\left(\xi_{x} \mathbf{F}+\xi_{y} \mathbf{G}\right), \quad \hat{\mathbf{G}}=\frac{1}{J}\left(\eta_{x} \mathbf{F}+\eta_{y} \mathbf{G}\right), \\
& \mathbf{U}=\left[\begin{array}{c}
\rho \\
\rho u \\
\rho v \\
\rho e
\end{array}\right], \mathbf{F}=\left[\begin{array}{c}
\rho u \\
\rho u^{2}+p \\
\rho v u \\
u(\rho e+p)
\end{array}\right], \mathbf{G}=\left[\begin{array}{c}
\rho v \\
\rho v u \\
\rho v^{2}+p \\
v(\rho e+p)
\end{array}\right], \\
& \hat{\mathbf{F}}_{v}=\frac{1}{J}\left[\begin{array}{c}
0 \\
\xi_{x_{i}} \tau_{i 1} \\
\xi_{x_{i}} \tau_{i 2} \\
\xi_{x_{i}}\left(u_{j} \tau_{i j}-q_{i}\right)
\end{array}\right], \mathbf{G}_{v}=\frac{1}{J}\left[\begin{array}{c}
0 \\
\eta_{x_{i}} \tau_{i 1} \\
\eta_{x_{i}} \tau_{i 2} \\
\eta_{x_{i}}\left(u_{j} \tau_{i j}-q_{i}\right)
\end{array}\right] \\
& \tau_{i j}=\frac{\mu}{R e_{\infty}}\left(\frac{\partial \xi_{k}}{\partial x_{j}} \frac{\partial u_{i}}{\partial \xi_{k}}+\frac{\partial \xi_{k}}{\partial x_{i}} \frac{\partial u_{j}}{\partial \xi_{k}}-\frac{2}{3} \delta_{i j} \frac{\partial \xi_{l}}{\partial x_{k}} \frac{\partial u_{k}}{\partial \xi_{l}}\right) \\
& q_{i}=-\frac{\mu}{R e_{\infty} \operatorname{Pr}(\gamma-1) M_{\infty}^{2}} \frac{\partial \xi_{j}}{\partial x_{i}} \frac{\partial T}{\partial \xi_{j}}, \\
& {\left[\begin{array}{l}
u_{1} \\
u_{2}
\end{array}\right]=\left[\begin{array}{l}
u \\
v
\end{array}\right],\left[\begin{array}{l}
x_{1} \\
x_{2}
\end{array}\right]=\left[\begin{array}{l}
x \\
y
\end{array}\right],\left[\begin{array}{l}
\xi_{1} \\
\xi_{2}
\end{array}\right]=\left[\begin{array}{l}
\xi \\
\eta
\end{array}\right]}
\end{aligned}
$$

The variables $\rho, u, v, p$, and $e$ are the density, Cartesian velocity components, pressure, and total specific energy, respectively. All the length scales and dependent variables have been nondimensionalized by the orifice width $d$ and the corresponding reference values, respectively, except for $p$ which has been normalized by $\rho_{\infty} u_{\infty}^{2}$. The Sutherland law for the viscosity coefficient $\mu$ and the equation of state for a perfect gas

$$
p=(\gamma-1) \rho\left[e-\frac{1}{2}\left(u^{2}+v^{2}\right)\right]
$$

are used to close Eq. (6). The ratio of specific heats $\gamma$ is assumed to be 1.4. Note that the $\xi$ and $\eta$ coordinates in Eq. (6) do not depend on time, i.e., the 2-D Navier-Stokes equations are solved on a stationary grid.

The governing equations are closed with the following boundary conditions. A no-slip boundary condition for the velocity vector and a constant wall temperature are imposed on the wall surface

$$
\left.u\right|_{\text {wall }}=\left.v\right|_{\text {wall }}=0,\left.\quad T\right|_{\text {wall }}=T_{\infty}
$$


The Blasius profile is used to set the solution vector at the inflow. At the subsonic outflow boundary, a boundary condition for the pressure is imposed weakly. Characteristic conditions are applied at the upper boundary so that the vortex structures can leave the computational domain without producing perceptible spurious reflections. The unsteady flow inside the actuator cavity, generated by harmonic motion of the diaphragm, is modeled by using a new reduced-order model described in the next section.

\section{A Reduced-Order Model of Synthetic Jet Actuators}

A gap exists between the $0-\mathrm{D}$ models and the full 2-D/3-D models of a synthetic jet actuator. To combine the accuracy and conservation properties of the full numerical simulation methods with the efficiency of the simplified blowing/suction type boundary conditions, a new reduced-order model of a multidimensional synthetic jet actuator is proposed. In contrast to the methods available in the literature, the new approach uses a reduced-order model to approximate a $2-\mathrm{D}$ or $3-\mathrm{D}$ actuator. The multidimensional actuator is simulated by solving the time-dependent quasi-1-D Euler equations. The time-dependent quasi-1-D Euler equations can be written in the following conservation law form:

$$
\begin{aligned}
\frac{\partial \mathbf{U}}{\partial t}+\frac{\partial \mathbf{E}}{\partial y}+\mathbf{H}=0, & {\left[\begin{array}{l}
\rho \\
\rho v \\
\rho e
\end{array}\right], \quad \mathbf{E}=A\left[\begin{array}{l}
\rho v \\
\rho v^{2}+p \\
v(\rho e+p)
\end{array}\right], \mathbf{H}=-\left[\begin{array}{l}
0 \\
p \frac{\partial A}{\partial y} \\
0
\end{array}\right], }
\end{aligned}
$$

where $A$ is the cross-sectional area of the quasi-1-D actuator. It is assumed that $A$ is a continuously differentiable function that is independent of time, i.e., $A=A(y)$ (see Fig. 2).

To simulate the diaphragm dynamics, a time-dependent one-to-one coordinate transformation,

$$
\begin{aligned}
& \tau=t \\
& \zeta=\zeta(t, y),
\end{aligned}
$$

is employed to map a physical domain with the moving boundary onto a unit interval. Note that the $\zeta$ coordinate depends on time and, therefore, a moving mesh technique is applied to solve the quasi-1-D Euler equations. Because the 
frequency of diaphragm oscillations $\omega$ is a given quantity, the moving mesh can be generated analytically

$$
y(\zeta, \tau)=(1-\zeta)[L+a(1-\cos (\omega \tau))],
$$

where $y$ and $\zeta$ are physical and computational coordinates, respectively, $a$ and $\omega$ are the amplitude and frequency of diaphragm oscillation, and $L+a$ is a mean depth of the quasi-1-D synthetic jet actuator.

The quasi-1-D Euler equations in the time-dependent coordinate frame can be rewritten in conservation law form as

$$
\frac{\partial \overline{\mathbf{U}}}{\partial \tau}+\frac{\partial \overline{\mathbf{E}}}{\partial \zeta}+\overline{\mathbf{H}}=0
$$

where

$$
\overline{\mathbf{U}}=\frac{\mathbf{U}}{J}, \quad \overline{\mathbf{E}}=\frac{1}{J}\left(\zeta_{t} \mathbf{U}+\zeta_{y} \mathbf{E}\right), \quad \overline{\mathbf{H}}=\frac{\mathbf{H}}{J}, \quad J=\frac{1}{y_{\zeta}} .
$$

Diaphragm oscillations are forced by varying the position of the diaphragm $y(0, \tau)$ where the impermeable wall boundary condition is imposed. Because the deforming mesh Eq. (10) is given analytically, the diaphragm velocity can be calculated by differentiating Eq. (10) with respect to time to give

$$
v(0, \tau)=a \omega \sin (\omega \tau)
$$

It should be noted that the region near the jet exit requires special consideration. As shown in Ref. [11], the full numerical simulation of the actuator orifice region is crucial for accurate prediction of the interaction between the synthetic jet and the external boundary layer. This region is characterized by strong flow separation that cannot be described by the quasi-1-D Euler equations. To overcome this problem, the quasi-1-D actuator model is used only to simulate the flow inside the actuator cavity, while the small region near the actuator orifice is modeled by solving the 2-D unsteady Navier-Stokes equations. The new reduced-order model is shown schematically in Fig. 2. The region where the quasi-1-D Euler equations are used is bounded by the dashed line, whereas in the rest of the domain, the 2-D Navier-Stokes equations are applied. This approach allows us to accurately predict the interaction of the synthetic jet with the external boundary layer and to resolve vortices generated in the vicinity of the actuator orifice, while reducing the computational cost.

The low-dimensional actuator model has several advantages. First, this approach is fully conservative and provides conservation of mass, momentum, and energy. Second, the new quasi-1-D model is computationally much more 


\section{2-D Navier-Stokes equations}

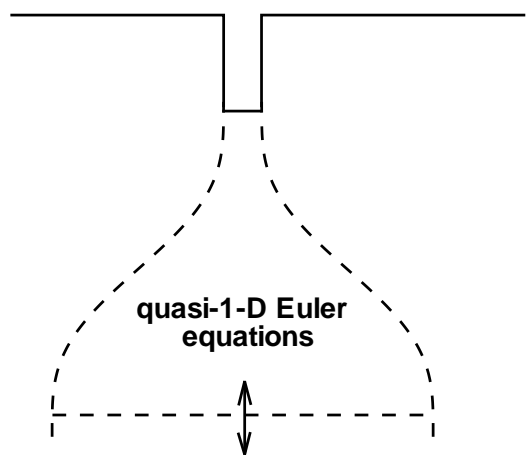

Fig. 2. A reduced-order model of a synthetic jet actuator.

efficient compared with the 2-D or 3-D numerical simulation of the cavity flow. Third, the reduced-order model retains some important multidimensional features of the realistic actuator, such as the length, diaphragm deflection, and area variation. These properties of the new model and its ability to account for the compressibility effects inherent in actuator devices make it an efficient tool for quantitative study of the actuator resonance characteristics.

The proposed reduced-order model is based on the assumption that the multidimensional and viscous effects inside the actuator cavity away from the orifice exit can be neglected. The detailed validation of the above assumption for a broad range of flow parameters and actuator geometries is presented in Section 5 .

\section{Fourth-Order Numerical Method}

A fourth-order upwind-biased linear finite difference scheme based on the local Lax-Friedrichs flux splitting is used to discretize both the 2-D Navier-Stokes equations (Eq. (6)) and the quasi-1-D Euler equations (Eq. (11)). This scheme can be written in a semidiscrete form as

$$
\frac{d \hat{\mathbf{U}}}{d \tau}+\frac{1}{2}\left(D_{4 \xi}^{-} \hat{\mathbf{F}}^{+}+D_{4 \xi}^{+} \hat{\mathbf{F}}^{-}+D_{4 \eta}^{-} \hat{\mathbf{G}}^{+}+D_{4 \eta}^{+} \hat{\mathbf{G}}^{-}\right)=D_{4 \xi}^{c} \hat{\mathbf{F}}_{v}+D_{4 \eta}^{c} \hat{\mathbf{G}}_{v}
$$


where $D_{4 \xi}^{ \pm}, D_{4 \xi}^{c}$ and $D_{4 \eta}^{ \pm}, D_{4 \eta}^{c}$ are linear fourth-order finite difference operators in $\xi$ and $\eta$, respectively. The Lax-Friedrichs fluxes are given by

$$
\begin{aligned}
\hat{\mathbf{F}}^{ \pm} & =\hat{\mathbf{F}} \pm\left|\lambda_{\xi}^{\max }\right| \hat{\mathbf{U}} \\
\hat{\mathbf{G}}^{ \pm} & =\hat{\mathbf{G}} \pm\left|\lambda_{\eta}^{\max }\right| \hat{\mathbf{U}}
\end{aligned}
$$

where $\left|\lambda_{\xi}^{\max }\right|$ and $\left|\lambda_{\eta}^{\max }\right|$ are the local maximum values of the contravariant eigenvalues $|\hat{u}|+\hat{c}$ and $|\hat{v}|+\hat{c}$, accordingly.

In the present analysis, the following upwind-biased fourth-order spatial operators $D_{4}^{ \pm}$are used to discretize the inviscid fluxes:

$$
\begin{aligned}
& D_{4}^{-} f_{j}=\frac{1}{12 h}\left(-f_{j-3}+6 f_{j-2}-18 f_{j-1}+10 f_{j}+3 f_{j+1}\right) \\
& D_{4}^{+} f_{j}=\frac{1}{12 h}\left(-3 f_{j-1}-10 f_{j}+18 f_{j+1}-6 f_{j+2}+f_{j+3}\right),
\end{aligned}
$$

where $h$ is a grid spacing either in $\xi$ or in $\eta$. The viscous fluxes are approximated by a fourth-order central finite difference operator given by

$$
D_{4}^{c} f_{j}=\frac{1}{12 h}\left(f_{j-2}-8 f_{j-1}+8 f_{j+1}-f_{j+1}\right) .
$$

The third-order boundary closures used near the boundaries are the optimal stencils derived from nearest neighbor information, biased where possible in an upwind direction.

The interface, inflow, and outflow boundary conditions are imposed weakly. The weak formulation of the wall boundary condition requires too many grid points near the wall to satisfy the no-slip condition. Therefore, the no-slip boundary condition for the velocity vector is imposed in the strong sense, which considerably decreases the number of grid points in the boundary layer while preserving the overall accuracy of the numerical solution. To satisfy the conservation laws at the interface between the 2-D and quasi-1-D domains, the $2-\mathrm{D}$ vector of conservative variables is "one-dimensionalized" as follows:

$$
\overline{\mathbf{U}}=\int_{0}^{d} \mathbf{U}(x, 0) d x .
$$

The above vector and the solution of the quasi-1-D Euler equations at the interface are then used as the left and right states of the Riemann problem which is solved approximately. The weak formulation of the interface boundary condition provides the conservation of mass, momentum, and energy and allows the ambient fluid to be entrained into the cavity and expelled via the jet. 
The quasi-1-D Euler and 2-D Navier-Stokes equations are integrated in time by using a five-stage, low-storage, explicit Runge-Kutta method developed in Ref. [17]. Rewriting the governing equations as an initial value problem

$$
\frac{\partial \mathbf{U}}{\partial t}=R H S(\mathbf{U})
$$

the low-storage explicit Runge-Kutta method is implemented as follows:

$$
\begin{aligned}
& \mathbf{U}^{1}=\mathbf{U}^{n} \\
& \mathbf{d U}^{l}=a_{l} \mathbf{d} \mathbf{U}^{l-1}+R H S\left(\mathbf{U}^{l-1}\right), \quad l=\overline{2,5} \\
& \mathbf{U}^{l}=\mathbf{U}^{l-1}+b_{l} \mathbf{d} \mathbf{U}^{l} \\
& \mathbf{U}^{n+1}=\mathbf{U}^{5},
\end{aligned}
$$

where $a_{l}$ and $b_{l}$ are functions of the standard Butcher coefficients, $\mathbf{U}^{n+1}$ is the fourth-order solution at time level $n+1$. The main advantage of this RungeKutta scheme is considerable reduction of computer memory usage because only two storage registers are required during the numerical integration.

\section{$5 \quad$ Numerical Results}

To evaluate accuracy and limitations of the new reduced-order model, the interaction between a synthetic jet actuator and a laminar boundary layer near a flat plate is used as a test problem. Standard atmospheric conditions are assumed for both freestream density and temperature. For all the test examples considered, the freestream Mach number is chosen to be 0.5 , which corresponds to a subsonic regime in the entire flowfield. Two actuator cavity shapes, box and nozzle-type geometries, are considered in the present analysis. For all the nozzle-type actuators considered, the geometry profile is given by the following 7 th-order polynomial:

$$
A(\bar{\zeta})=A_{r}+\left(A_{r}-A_{l}\right) \bar{\zeta}^{4}\left(-35+84 \bar{\zeta}-70 \bar{\zeta}^{2}+20 \bar{\zeta}^{3}\right)
$$

where $\bar{\zeta}=\left(y-y_{r}\right) /\left(y_{l}-y_{r}\right), A_{l}$ and $A_{r}$ are areas of the actuator diaphragm and orifice, respectively. For each test problem, 10 cycles of diaphragm oscillation are calculated to eliminate initial transients and to accumulate statistics.

Because the exact solution to this complicated time-dependent problem is not available, the accuracy of the reduced-order model is evaluated by comparing the numerical solutions obtained with the quasi-1-D model and the full 2-D Navier-Stokes simulation of the same actuator. To avoid the integration of the 2-D Navier-Stokes equations on a moving grid, the diaphragm dynamics 
is simulated by using the quasi-1-D Euler equations. In other words, the 2D Navier-Stokes equations are employed in the entire actuator cavity, except for a small region near the moving diaphragm that is modeled in a quasi-1D sense, as described in Section 3. This treatment of the moving diaphragm allows us to perform full 2-D numerical simulations without using a timedependent coordinate transformation. Because the region where the quasi-1-D Euler equations are used is much less than that described by the 2-D NavierStokes equations, this combined approach accounts for the multidimensional and viscous effects inside the actuator cavity and provides high accuracy of the numerical solution.

\subsection{Validation of the 0-D model}

Before validating the new reduced-order model of synthetic jet actuators, a conventional 0-D model is tested. To incorporate the 0-D model into the high-order finite difference formulation used in the present analysis, the blowing/suction boundary conditions Eqs. (1) and (4) are imposed in the weak sense. To be consistent with the 0-D model, the diaphragm deflection in the quasi-1-D and 2-D simulations is adjusted such that the peak mass rate at the orifice exit equals that prescribed in the boundary conditions Eqs. (1) and (4). In contrast to the 0-D models available in the literature, in the present analysis, the blowing/suction boundary conditions are imposed inside the actuator neck rather than at the orifice exit. This implementation of the boundary conditions makes the 0 - $\mathrm{D}$ model more accurate because the viscous effects near the jet exit are taken into account. It also makes the comparison between 0-D and quasi-1-D models more consistent because the boundary conditions are imposed along the same line where the interface between the quasi-1-D and 2 -D models is located.

Table 1

\begin{tabular}{|c|c|c|}
\hline $\begin{array}{c}\text { Interface location } \\
\text { (Distance from the surface) }\end{array}$ & $\begin{array}{c}\text { 0-D model } \\
\left(\text { Mass } \times 10^{2}\right)\end{array}$ & $\begin{array}{c}\text { Quasi-1-D model } \\
\left(\text { Mass } \times 10^{2}\right)\end{array}$ \\
\hline 2 & 0.729 & 0.0622 \\
5 & 1.326 & 0.0113 \\
10 & 2.046 & 0.0902 \\
\hline
\end{tabular}

The total mass injection over a period of diaphragm oscillation.

One distinctive feature of synthetic jet actuators is that the net mass injection into the overall system over each cycle of operation is zero. This characteristic feature of synthetic jet actuators is used to estimate accuracy of the 0-D and 


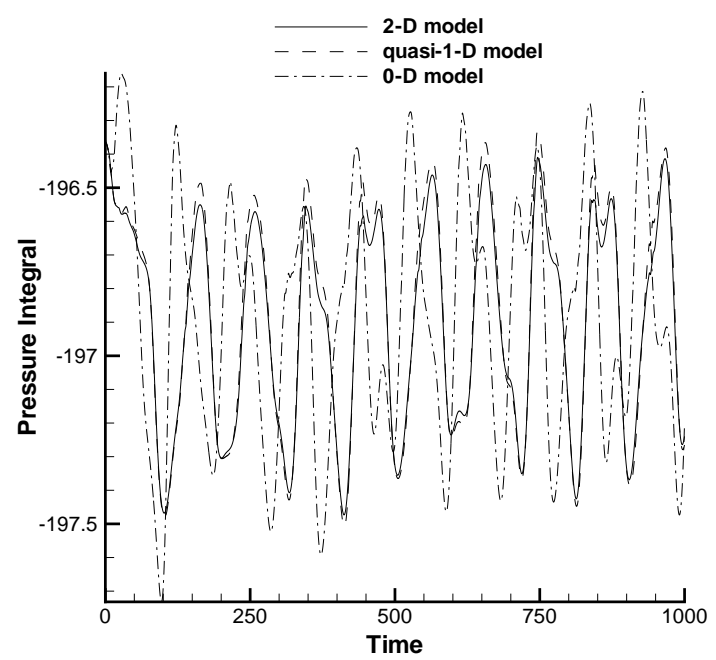

Fig. 3. Time histories of the pressure integral obtained with the 0-D, quasi-1-D, and 2-D models.

quasi-1-D models. Three calculations corresponding to different locations of the interface between the low-dimensional model and the full 2-D model are performed. The computational parameters are as follows: $R e_{d}=500, A_{l}=$ $10, L=20, \omega=0.1257$, and $a=0.2387$. The total mass injection over a period of diaphragm oscillation, obtained with the 0-D and quasi-1-D models, is presented in Table 1. As follows from these results, the quasi-1-D model is one order of magnitude more accurate than the 0 -D counterpart. This result is not surprising because the blowing/suction boundary conditions do not satisfy the conservation laws, whereas the new reduced-order model is based on the conservation laws equations.

Another example demonstrating the superiority of the quasi-1-D model over the 0-D models is shown in Fig. 3. As seen in the figure, the pressure integrals obtained with the quasi-1-D and full 2-D models are practically indistinguishable from each other, whereas the 0 -D model results are completely inaccurate. The lack of accuracy of the 0-D model is due to the heuristic assumptions about the velocity profile and pressure gradient that have been made to construct the simplified boundary conditions given by Eqs. (1) and (4).

\subsection{Validation of the quasi-1-D model}

To validate the new reduced-order model, the synthetic jet generated by a nozzle-type actuator with a long $10 d$ neck is simulated by using both the new quasi-1-D and 2-D models. Figure 4 shows a 4-block structured computational grid having $280 \times 100$ cells in the exterior region and $80 \times 200$ cells inside 


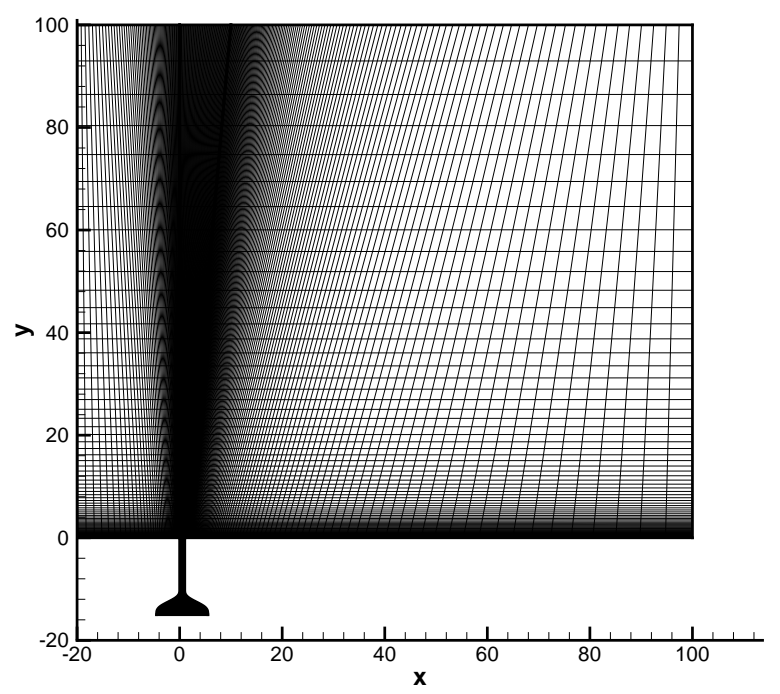

Fig. 4. Computational grid.

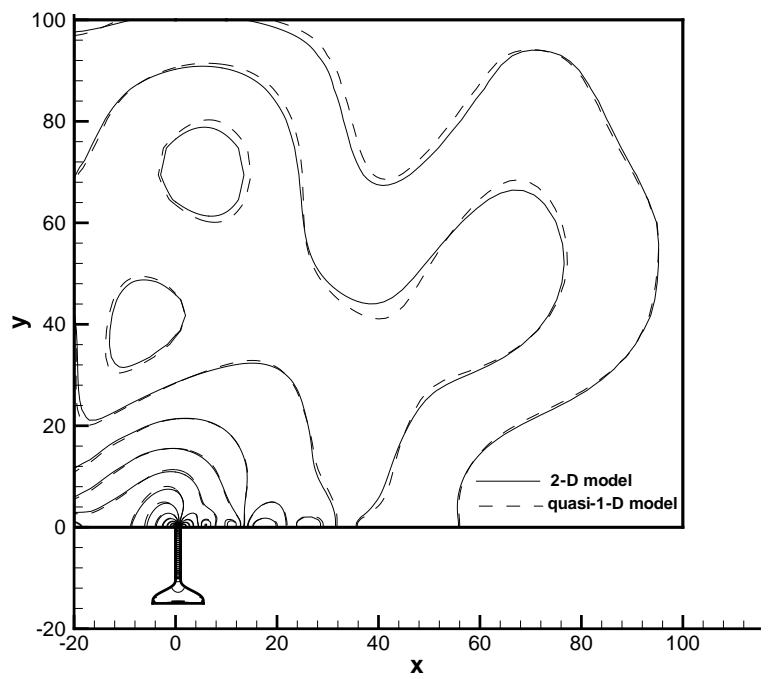

Fig. 5. Instantaneous pressure contours obtained with the quasi-1-D and 2-D models at time $T=500$.

the actuator cavity. The grid points are clustered along the solid surfaces such that about 20 grid points are located inside the boundary layer. The Reynolds number, diaphragm deflection, nondimensional width, and depth of the actuator cavity are $500,0.2387,10$, and 16 , respectively.

Snapshots of pressure contours obtained with both the quasi-1-D and 2-D models after 10 cycles of diaphragm oscillation are presented in Fig. 5. Although the quasi-1-D model does not model the boundary layer and its separation inside the actuator cavity, the conservation properties of the new model and the detailed simulation of the flow recirculation near the slot exit provide practically the same accuracy as the full 2-D model. 


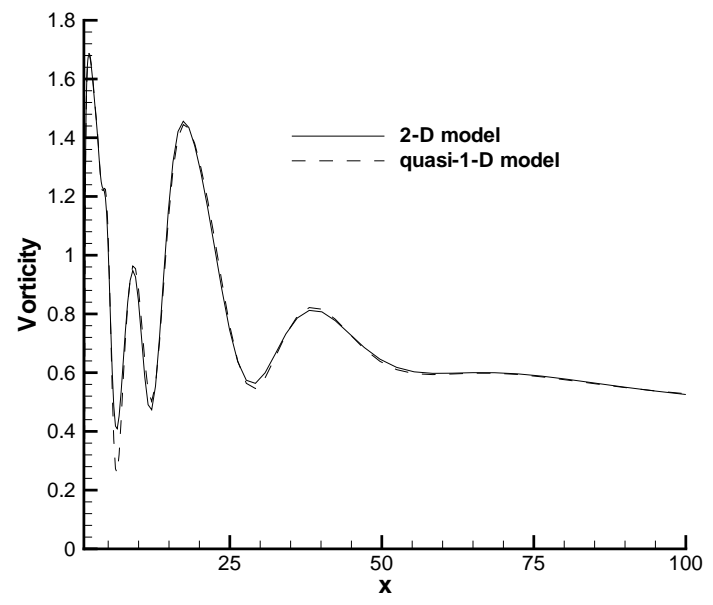

Fig. 6. Instantaneous vorticity distributions obtained with the quasi-1-D and 2-D models at time $T=500$.

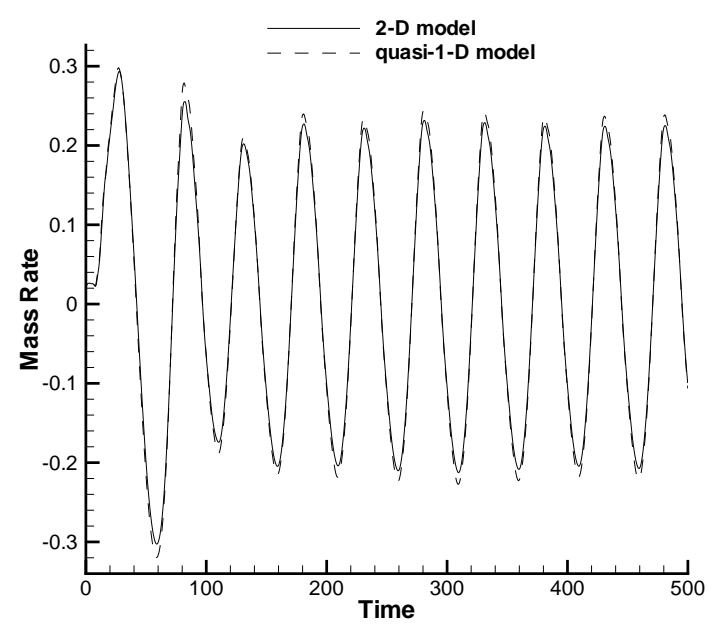

Fig. 7. Time histories of the jet mass rate at the orifice exit obtained with the quasi-1-D and 2-D models.

The instantaneous vorticity distributions on the flat plate wall downstream of the actuator slot, calculated by using the quasi-1-D and 2-D models, is shown in Fig. 6. The solution obtained with the reduced order model is almost indistinguishable from the corresponding full 2-D numerical simulation results, except for the local extremum located near the downstream corner of the actuator slot. This difference is due to the proximity of the orifice corner, which is a geometrically singular point.

To show how accurately the new quasi-1-D actuator model predicts the synthetic jet characteristics, Fig. 7 presents the jet mass rates at the orifice exit, obtained with the reduced-order and full 2-D models. Both solutions become 


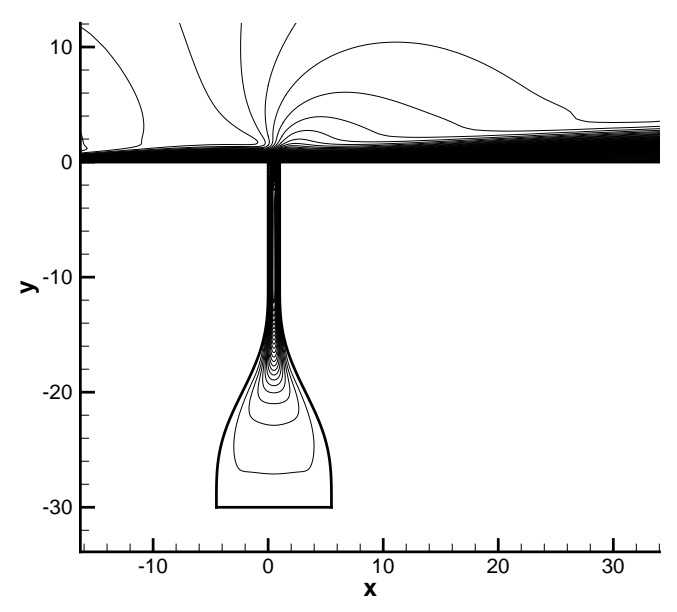

Fig. 8. Instantaneous Mach number contours obtained with the full 2-D model at $R e_{d}=200$.

practically periodic after five cycles of diaphragm oscillation. The maximum deviation between the quasi-1-D and 2-D solutions, which occurs at the peaks, is less than $3 \%$. The reduced-order model slightly overpredicts the peak values of the mass rate because the quasi-1-D Euler equations do not account for the viscous losses inside the actuator cavity. The results presented in Fig. 7 indicate that the multidimensional and viscous phenomena associated with the boundary layer and its separation inside the actuator cavity away from the orifice have almost no effect on either the synthetic jet characteristics or the exterior flow.

\subsection{Sensitivity of the quasi-1-D model to Reynolds number}

As mentioned in Section 3, the new reduced-order model does not account for the viscous losses that occur inside the actuator cavity. To evaluate the effect of viscosity on accuracy of the quasi-1-D model of a nozzle-type actuator, three calculations at Reynolds numbers 200, 500, and 1000 are performed. For all three calculations, the cavity depth, amplitude and frequency of diaphragm oscillation are 30, 0.4775 , and 0.0628 , respectively. At $R e_{d}=200$, the viscous effects are predominant. Figure 8 shows that the boundary layer rapidly grows and becomes fully developed inside the actuator neck. The growth of the boundary layer restricts the mass flow through the actuator orifice. The result is that the synthetic jet mass rate predicted by the quasi-1-D model is about $20 \%$ larger than that obtained with the full 2-D Navier-Stokes simulation of the cavity flow, as seen in Fig. 9. As the Reynolds number increases, the viscous effects inside the actuator cavity become weaker, leading to a better agreement between the quasi-1-D and 2-D models. As expected, the quasi- 

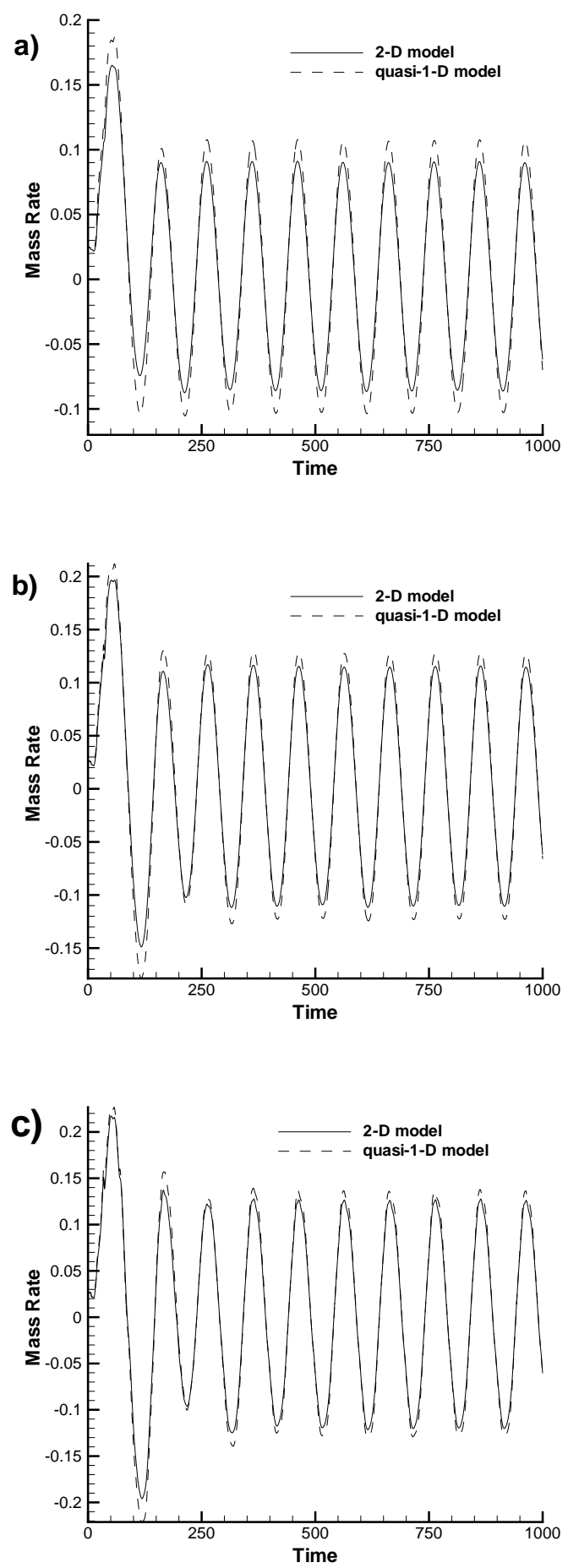

Fig. 9. Time histories of the jet mass rate obtained with the quasi-1-D and 2-D models at: a) $R e_{d}=200$, b) $R e_{d}=500$, and c) $R e_{d}=1000$.

1-D model provides the highest accuracy for the largest Reynolds number considered. For $R e_{d}=500$ and $R e_{d}=1000$, the synthetic jet mass rates obtained with the quasi-1-D and 2-D models during the periodic regime agree 


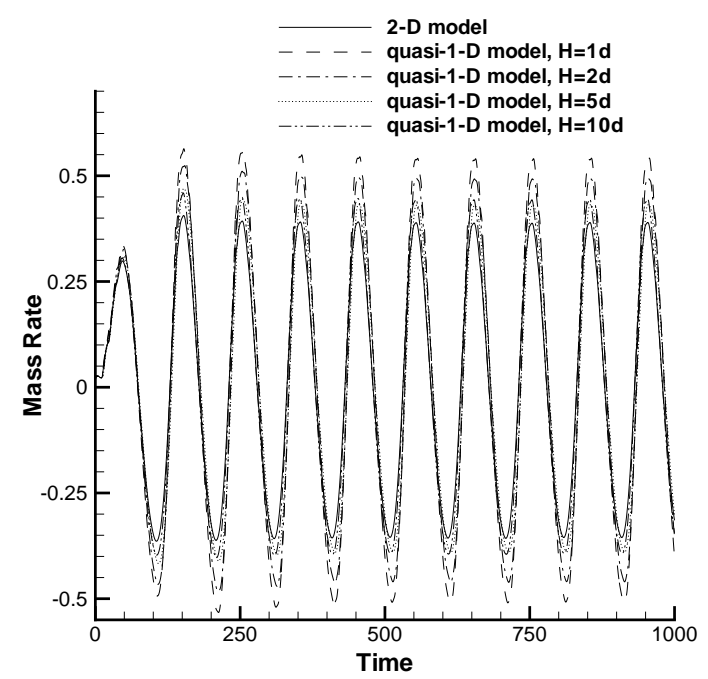

Fig. 10. Effect of the interface location on accuracy of the quasi-1-D model.

to within 5-7\%. Based on the results presented in Fig. 9, we conclude that the new reduced-order model accurately predicts the synthetic jet characteristics of this geometry for $R e_{d} \geq 500$.

\subsection{Sensitivity of the quasi-1-D model to interface location}

Another issue that requires special investigation is sensitivity of the new reduced-order model to a location of the interface between the quasi-1-D Euler equations and the 2-D Navier-Stokes equations. As mentioned previously, the quasi-1-D model is not valid near the orifice exit where the strong boundary layer separation occurs, which should be simulated by using the full 2-D Navier-Stokes equations. To quantify the influence of interface location on the overall numerical solution accuracy, four calculations with the interface located $1 d, 2 d, 5 d$, and $10 d$ away from the orifice exit are carried out. The Reynolds number and the cavity depth are chosen to be 500 and 20, respectively; the diaphragm deflection and frequency are equal to those used in the foregoing test problem. Figure 10 shows the effect of interface location on the predicted temporal variation of the synthetic jet mass rate at the orifice exit. The closer the interface is located to the flat plate surface, the less accurate the quasi-1-D model becomes. The main reason for such a behavior is the inability of the quasi-1-D Euler equations to describe the flow recirculation near the jet exit. However, if the interface is located more than $2 d$ away from the orifice exit, the agreement between the quasi-1-D model and the 2-D full Navier-Stokes simulation is good. These results suggest that only the small region near the jet exit, which is comparable with the actuator slot size, should be simulated by using the 2-D Navier-Stokes equations, whereas the rest of the cavity flow can be accurately predicted by the quasi-1-D Euler equations. 


\section{2-D Navier-Stokes equations}

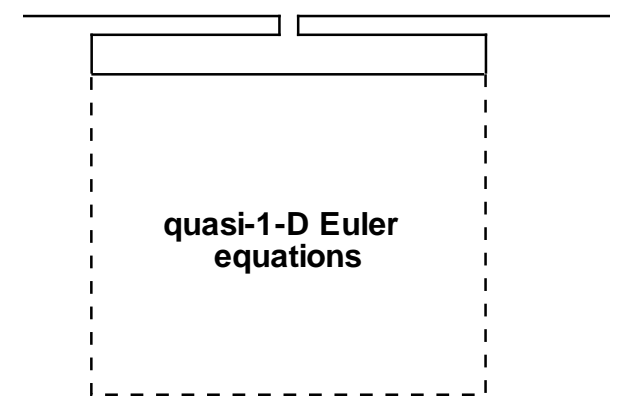

Fig. 11. Application of the quasi-1-D model to a box actuator.

\subsection{Sensitivity of the quasi-1-D model to actuator geometry}

The results presented so far were obtained for the nozzle-type actuators with a long neck. To test the sensitivity of the new quasi-1-D model to actuator geometry, a $20 d \times 20 d$ box actuator with a very short $1 d$ neck is considered. The calculation is performed at $R e_{d}=1000, \omega=0.1257$, and $a=0.2387$. In contrast to the nozzle-type profile, inside the actuator cavity, the box geometry has sharp corners that cannot be accurately approximated by a smooth area variation function $A(y)$. Note that the quasi-1-D Euler equations are derived under the assumption that the area $A(y)$ is a continuously differentiable function. Furthermore, these sharp corners cause the strong flow separation and generate vortices inside the actuator cavity during the expulsion and ingestion strokes. These conditions indicate that not only the actuator neck but also a small portion of the actuator cavity near the jet exit should be simulated by using the 2-D Navier-Stokes equations. To overcome this problem and simultaneously reduce the computational cost, the quasi-1-D Euler equations are used to solve $90 \%$ of the cavity flow; the remaining actuator flowfield near the orifice exit is described by the 2-D Navier-stokes equations, as shown schematically in Fig. 11.

To demonstrate the effect of actuator geometry on accuracy of the new reducedorder model, Fig. 12 shows a time history of the pressure integral measured on the flat plate wall downstream of the slot exit, which is obtained with the quasi-1-D model, along with the full 2-D Navier-Stokes simulation. Although the quasi-1-D Euler equations do not describe the complex behavior of vortex structures inside the actuator cavity, the agreement between the reducedorder model and the full 2-D simulation in the exterior flowfield is excellent. In contrast to the 0-D models, the new quasi-1-D model does not require any information about the velocity profile at the jet exit. This property of the new 


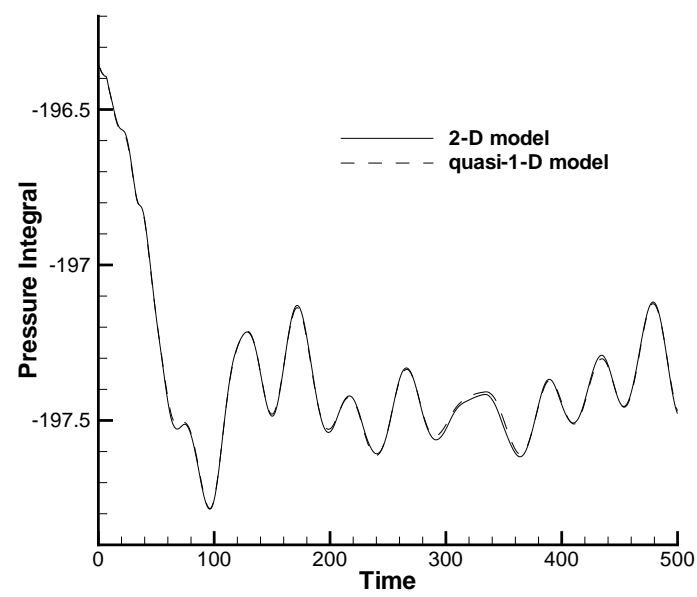

Fig. 12. Time histories of the pressure integral obtained with the quasi-1-D and 2-D models for the box actuator.

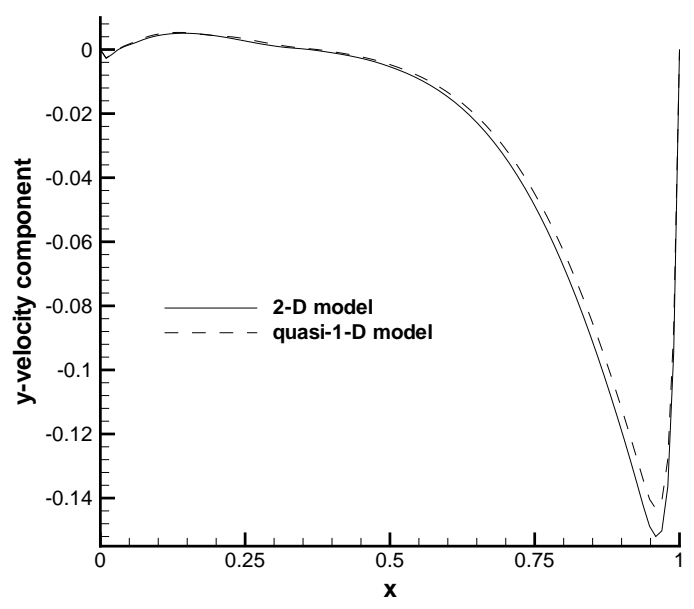

Fig. 13. Instantaneous velocity profiles at the orifice exit of the box actuator, obtained with the quasi-1-D and 2-D models.

model is demonstrated in Fig. 13, which shows the instantaneous jet velocity profiles measured at the slot exit after 10 cycles of diaphragm oscillation. The jet velocity profile is essentially asymmetric with a peak near the downstream edge of the orifice exit and resembles neither the top-hat nor the sinusoidal profiles of Kral et al. [2]. Although the multidimensional effects are essential near the orifice exit, the results obtained with the quasi-1-D model are in a very good agreement with that of the full $2-\mathrm{D}$ model. 


\subsection{Resonance characteristics of synthetic jet actuators}

It is well-known that resonance effects play a crucial role in improvement of efficiency and performance of synthetic jet actuator devices. From this perspective, it is important to be able to predict and optimize the resonance characteristics of synthetic jet actuators. To demonstrate the ability of the new reduced-order model to simulate the acoustic resonance inherent in synthetic jet actuators, two similar problems are solved. In the first problem, an isolated nozzle-type synthetic jet actuator placed in a quiescent flow is simulated by the fully inviscid quasi-1-D model. The exterior flowfield is also modeled in a quasi-1-D sense by prescribing appropriate boundary conditions at the quasi-1-D actuator orifice exit. The second problem considers the interaction of the same synthetic jet actuator with a crossflow. The quasi-1-D actuator model is employed to simulate the cavity flow, whereas the exterior crossflow and the small region near the jet exit are modeled by using the 2-D Navier-Stokes equations. In both cases, we perform a series of calculations at different frequencies, which allow us to quantify the influence of the crossflow and the viscous effects on the actuator resonance characteristics.

Figure 14 shows the peak mass rates at the orifice exit after 10 cycles of diaphragm oscillation, obtained for both cases. Note that each point in each curve corresponds to a separate calculation at a different frequency, and only the peak value of the mass rate obtained in each run is shown in the figure. The diaphragm amplitude and frequency are chosen such that the peak mass rate at the diaphragm remains unchanged for all the numerical calculations presented.

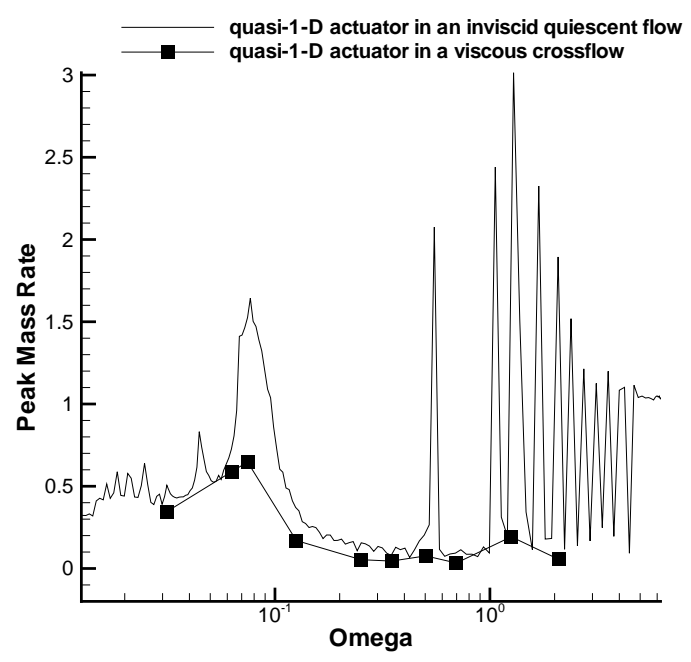

Fig. 14. Effect of the actuator/boundary layer interaction on the resonance characteristics of the synthetic jet actuator. 
Three conclusions can be drawn from this comparison. First, the results presented in Fig. 14 demonstrate that although the diaphragm pushes the same amount of fluid for all the frequencies considered, the jet velocity strongly depends on the diaphragm frequency. The main reason for such a behavior is fluid compressibility. In both cases, the peak mass rate at the orifice exit exhibits resonance behavior similar to the Helmholtz resonance inherent in cavity flows. If the diaphragm frequency differs from its resonance value $\omega=0.077$, the peak mass rate drastically decreases. We emphasize that in both cases, the resonance mass rate at the orifice exit is more than one order of magnitude larger than its minimum value. In other words, the effectiveness of the synthetic jet actuator can be tremendously improved by optimizing the diaphragm frequency.

Second, the viscous effects considerably decrease the mass rate amplitude at the orifice exit. Because the Reynolds number based on the slot width was set to 500, the viscosity effects associated with the external boundary layer/synthetic jet interaction and growth of the viscous layer in the actuator orifice are dominant and restrict the mass flow through the orifice. As a result, the resonance mass rate amplitude at the orifice exit decreases by a factor of two compared with that obtained with the fully quasi-1-D Euler simulation of the isolated actuator.

Third, the presence of the crossflow and viscosity have almost no effect on the resonance frequency of the synthetic jet actuator. Although the fully developed boundary layer and its separation near the jet exit significantly reduce the mass rate amplitude, the resonances obtained in both cases occur at the same frequency. These results indicate that the inviscid quasi-1-D model can be used to quantitatively predict the resonance frequency of realistic synthetic jet actuators.

\section{Discussion}

The broad objective of this study is to present proof of concept for representing realistic fluid actuators by reduced-order models based on a quasi-1-D approach. Encouraging results are obtained for all geometries studied. Inevitably, however, some geometries will not be well represented in quasi-1-D form. Likely candidates include 3-D actuators that have two or more dominant Helmholtz resonance length-scales as opposed to a single dominant scale. A precise statement about the absolute generality of this approach is not currently possible, but this issue is the focus of ongoing work.

Two different geometries are the primary focus of the present study. The first consists of a nozzle-type synthetic jet actuator with a long neck (neck to orifice 
ratio of 10:1). The second geometry is a box-type symmetric actuator with a short neck (neck to orifice ratio of 1:1). Exact comparisons are made between the quasi-1-D approach and full 2-D simulations in both cases, thus allowing a quantitative measure of error. Geometries that did not allow for an "exact" solution (full 2-D simulation) were not considered.

The exact shape and characteristics of realistic actuators continue to evolve with different applications. The shape of actual devices will likely lie between the two extremes presented in this work and will be amenable to the quasi1-D model. In this respect, it is important to determine how to optimize actuator parameters (size, geometry, and diaphragm frequency) to provide the maximum performance (mass rate at the orifice exit). The best candidate for solving this optimization problem is the new reduced-order model because of its efficiency and accuracy.

Ongoing work continues to determine the generality of the quasi-1-D representation for complex devices. It is critical to determine which 3-D resonator shapes are not well suited for the quasi-1-D approach. If secondary harmonics are unimportant (moderate accuracy tolerances), perhaps it is possible to model the primary harmonic of a 3-D device by an effective quasi-1-D geometry. Another outstanding issue is the fidelity required in the diaphragm region to achieve acceptable levels of global accuracy. Actuator diaphragms can undergo structural resonances that are frequency-related and could interact with the acoustic modes.

Finally, a careful study of the sensitivity of these results to external conditions is required. Nondimensional parameters that influence the overall performance are the actuator Reynolds, Mach, and Stokes numbers, as well as the external Reynolds number (boundary layer thickness). Simple scaling arguments suggest that external vorticity can be ingested by the actuator if the eddy length-scales are small compared with the actuator orifice. Ingested vortices could affect the resonance characteristics of the device.

\section{Conclusions}

The new reduced-order model of a synthetic jet actuator has been proposed and validated. The main idea of the new approach is to use the low dimensional model of 2-D/3-D synthetic jet actuators, which is based on the quasi-1-D Euler equations. The oscillating diaphragm is simulated as a quasi-1-D moving boundary. The new reduced-order model possesses the following important characteristics. First, it satisfies the conservation laws. Also, the new reducedorder model is efficient in terms of computational time because only the 1-D equations need to be solved. The quasi-1-D model retains important multi- 
dimensional features of the realistic actuator governed by the length, area variation, diaphragm deflection, and frequency and can therefore be used for predicting the synthetic jet dynamics and the actuator resonance characteristics. The proposed reduced-order model is flexible and can be used for a broad class of actuator geometries ranging from smooth nozzle-type to nonsmooth box-type profiles. Furthermore, the fluid/structure interaction can be included into the new quasi-1-D actuator model by adding the terms associated with the structural stiffness of the actuator membrane. Because the actuator is simulated in a quasi-1-D sense, the new model does not take into account the multidimensional effects associated with the vortex generation, boundary layer and its separation inside the actuator cavity. However, quantitative comparison of the quasi-1-D and full 2-D models shows that if the Reynolds number based on the actuator slot width is larger than 500 and the interface between the quasi-1-D Euler equations and the 2-D Navier-Stokes equations is located more than $2 d$ away from the jet exit, then the new reduced-order model provides high accuracy in the exterior flowfield. Another important conclusion is that only the multidimensional effects near the jet exit are essential: these should be simulated by using the full Navier-Stokes equations, whereas the cavity flow can be accurately predicted by the quasi-1-D Euler equations. One of the main advantages of the new reduced-order model is its ability to predict the acoustic resonance inherent in synthetic jet actuators. As follows from the numerical study of the actuator resonance characteristics, the synthetic jet mass rate at the orifice exit can be increased by more than one order of magnitude by moving the diaphragm at the acoustic resonance frequency. The developed reduced-order model opens new avenues for the detailed study of synthetic jet actuators and optimization of their performance, which is not feasible by using the existing methodologies based on either the $0-\mathrm{D}$ or $3-\mathrm{D}$ model.

\section{References}

[1] B. L. Smith and A. Glezer, Vectoring and small-scale motions effected in free shear flows using synthetic jet actuators, AIAA Paper 97-0213, 1997.

[2] L. D. Kral, J. F. Donovan, A. B. Cain, and A. W. Cary, Numerical simulation of synthetic jet actuators, AIAA Paper 97-1824, 1997.

[3] J. F. Donovan, L. D. Kral, and A. W. Cary, Active flow control applied to an airfoil, AIAA Paper 98-0210, 1998.

[4] A. Hassan and R. D. JanakiRam, Effects of zero-mass "synthetic" jets on the aerodynamics of the NACA-0012 airfoil, J. Amer. Heli. Soc., Vol. 43, 1998, pp. 303-311.

[5] H. A. Carlson and J. L. Lumley, Flow over an obstacle emerging from the wall of a channel, AIAA J., Vol. 34, 1996, pp. 924-931. 
[6] L. M. Hoffmann and T. Herbert, Disturbances produced by the motion of an actuator, Phys. of Fluids, Vol. 9, 1997, pp. 3727-3732.

[7] H. Lin and C. C. Chieng, Computations of compressible synthetic jet flows using multigrid/dual time stepping algorithm, AIAA Paper 99-3114, 1999.

[8] P. W. Carpenter, D. A. Lockerby, and C. Davies, Numerical simulation of the interaction of MEMS actuators and boundary layers, AIAA Paper 2000-4330, 2000 .

[9] R. Rathnasingham and K. S. Breuer, Characteristics of resonant actuators for flow control, AIAA Paper 96-0311, 1996.

[10] Q. Gallas, J. Mathew, A. Kaysap, R. Holman, T. Nishida, B. Carrol, M. Sheplak, and L. Cattafesta, Lumped element modeling of piezolectric-driven synthetic jet actuators, AIAA Paper 2002-0125, 2002.

[11] D. P. Rizzetta, M. P. Visbal, and M. J. Stanek, Numerical investigation of synthetic jet flowfields, AIAA Paper 98-2910, 1998.

[12] R. D. Joslin, J. T. Lachowicz, and C. S. Yao, DNS of flow induced by a multiflow actuator, ASME FEDSM 98-5302, Proc. of the ASME Fluids Engineering Conf., Forum on Control of Transitional and Turbulent Flows, ed. D. E. Parekh, Washington D.C., 1998.

[13] R. Mittel, P. Ramunggoon, and H. S. Udaykumar, Interaction of a synthetic jet with a flat plate boundary layer, AIAA Paper 2001-2773, 2001.

[14] C. Y. Lee and D. B. Goldstein, Two-dimensional synthetic jet simulation, AIAA Paper 2000-0406, 2000.

[15] A. Seifert, T. Bachar, D. Koss, M. Shepshelovich, and I. Wyganski, Oscillatory blowing: A toll to delay boundary-layer separation, AIAA J., Vol. 31, No. 11, 1993, pp. 2052-2060.

[16] A. Seifert, A. Darabi, and I. Wyganski, Delay of airfoil stall by periodic excitation, AIAA J. Aircr, Vol. 33, No. 4, 1996, pp. 691-698.

[17] C. A. Kennedy, M. H. Carpenter, and R. Lewis, Low-storage, explicit RungeKutta schemes for the compressible Navier-Stokes equations, Appl. Numer. Math., Vol. 35(3), 2000, pp. 177-219. 


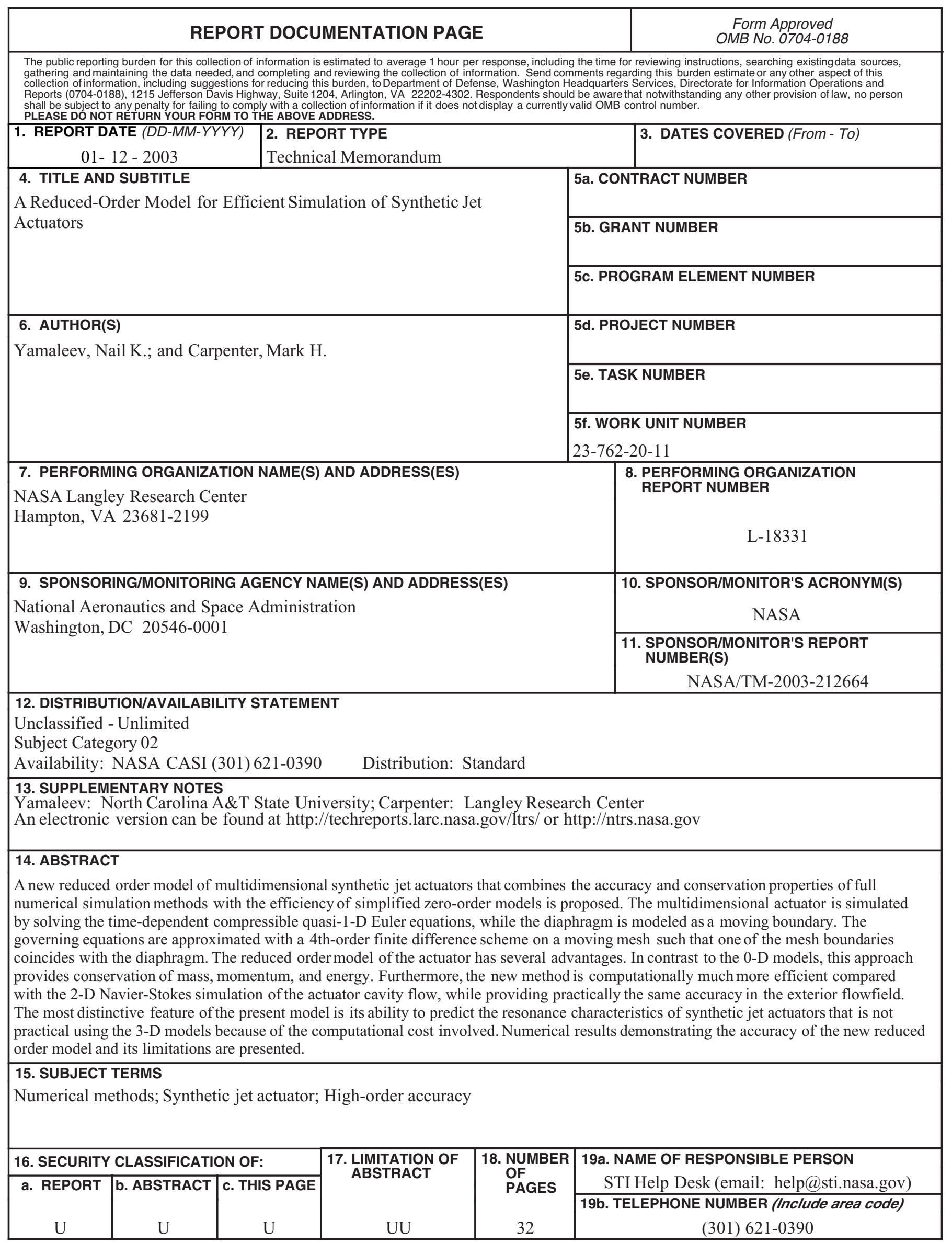

